\title{
Diferencias de sexo, edad y lípidos plasmáticos asociadas al polimorfismo de la apolipoproteína E en un grupo de escolares de Quindío, Colombia
}

\author{
Patricia Landázuri', Nelsy Loango ${ }^{1,2}$, Martha Lucía Gallego ${ }^{1}$, Beatriz Restrepo $^{1}$ \\ 1 Grupo de Enfermedades Cardiovasculares y Metabólicas, Facultad de Ciencias de la Salud, \\ Universidad del Quindío, Armenia, Colombia \\ 2 Programa de Biología, Facultad de Ciencias Básicas y Tecnologías, Universidad del Quindío, \\ Armenia, Colombia
}

Introducción. El polimorfismo de la apolipoproteína $E$ (apoE) es un factor determinante genético de los niveles de colesterol total y colesterol LDL.

Objetivo. Evaluar la relación del polimorfismo apoE con el perfil lipídico por edad y sexo en escolares quindianos de 8 a18 años.

Materiales y métodos. Se utilizó la reacción en cadena de la polimerasa y enzimas de restricción para la genotipificación de apoE en 500 escolares de 8 a 18 años. Los lípidos sanguíneos se determinaron con estuches comerciales.

Resultados. La frecuencia alélica fue: E3 (91,6\%), E2 (5,3\%) y E4 (3,1\%); y la frecuencia genotípica: E3/E3 (90,8\%), E2/E3 (5,6\%) y E3/E4 (3,2\%). No se encontraron homocigotos para E2/E2 y E4/E4. Se encontraron distribución genotípica y alélica similares para cada sexo.

El genotipo apoE y el sexo tuvieron un efecto significativo sobre el colesterol total y el colesterol LDL. En niños, ambas variables se distribuyeron así: E4>E3>E2 $(p<0,05)$; en niñas: E3>E4>E2; el colesterol LDL en niños fue: E2>E4>E3; y, en niñas: E4>E2>E3.

En niños, el alelo con efecto protector fue el E2, en todas las variables; mientras que, en las niñas, este alelo fue protector sólo para el colesterol total y colesterol LDL; para triacilglicéridos y colesterol VLDL y altos niveles de colesterol LDL, el efecto protector estuvo en el $E 4(p<0,05)$.

Conclusión. Estos resultados sugieren una asociación entre el genotipo apoE y los lípidos plasmáticos de la población estudiada, influenciada por sexo y edad, especialmente, en niñas mayores de 10 años. Sin embargo, se necesita ampliar la población.

Palabras clave: polimorfismo genético, apolipoproteínas E, lípidos, colesterol, triglicéridos, polimorfismo, hormonas, niño, adolescente.

Gender, age and plasma lipids differences associated with apolipoprotein E polymorphism in school children

Introduction. Apolipoprotein $\mathrm{E}$ (apoE) polymorphism is a genetic determinant of total cholesterol and LDL-c levels. Several studies have examined the relationship between variations at the APO $\mathrm{E}$ and cardiovascular disease. It is important to determine this relationship in the Colombian population.

Objectives. The relationship of apoE polymorphisms was associated with lipid profile by age and sex.

Materials and methods. A sample of 500 children aged 8 to 18 years, were selected from school populations in Quindio Province, Colombia. ApoE genotypes were determined by polymerase chain reaction and restriction enzyme. Lipid profiles were obtained by commercial kits.

Results. The apoE allele frequencies were as follows: E3 - 91.6\%, E2 - 5.3, and E4 - 3.1\%. Genotypic frequencies were as follows: E3/E3 - 90.8\%, E2/E3 - 5.6\%, and E3/E4 - 3.2\%. No homozygotes for E2/E2 and E4/E4 were recovered. Similar genotypic and allelic distribution was found for each gender. ApoE genotype and gender had a significant effect on total serum cholesterol (CT) and low density lipoprotein cholesterol (c-LDL). In boys these variables were related as follows: E4>E3>E2 $(p<0.05)$; in girls the relationships were somewhat changed 
(E3>E4>E2). High density lipoprotein cholesterol (c-HDL) levels in boys were related as follows: E2>E4>E3; for girls, E4>E2>E3. In boys, for all variables, the protector effect was in E2; but in girls this allele was only a protector for CT and c-LDL, For triacylglycerol (TAG), very low density lipoprotein cholesterol (c-VLDL) and c-HDL, this the protector effect was associated with the E4 allele, $(\mathrm{p}<0.05)$.

Conclusion. The results suggested that modulation of apoE genotype on plasma lipids was influenced by gender and age, especially in girls older than 10 years.

Key words: Polymorphism, genetic; apolipoproteins E, lipids, cholesterol, triglycerides, hormones, child, adolescent.

El polimorfismo de la apoE es un factor determinante genético de los niveles de colesterol total y colesterol en las lipoproteínas de baja densidad (c-LDL) en plasma y, por lo tanto, un factor determinante de las enfermedades cardiovasculares (1-3).

Se ha estimado que $14 \%$ de la variación plasmática del colesterol está determinada genéticamente por el polimorfismo de apoE (3), mediante la interacción de las isoformas con el receptor de las LDL, lo cual permite la depuración de estas lipoproteínas y, por lo tanto, la reducción del colesterol y los triglicéridos. Entre las mayores isoformas de la apoE, las cuales son designadas como apoE2, apoE3 y apoE4, la apoE3 es la más común en todas las poblaciones estudiadas, con una frecuencia en la población de $77 \%$ y se considera la forma normal de la proteína (3). Comparadas con la apoE3, la apoE2 está asociada con bajo colesterol total en plasma y, la apoE4, con altos niveles de colesterol total y c-LDL y, está última, con un aumento en la captación posprandial de las lipoproteínas, aumento de la absorción intestinal de colesterol, descenso en la síntesis de bilis y rápida depuración de las LDL del plasma $(3,4)$.

Las enfermedades cardiovasculares son la primera causa de mortalidad en el mundo y en Colombia $(5,6)$. En este país, algunos estudios de población muestran la presencia de un alto porcentaje de dislipidemias y obesidad en niños (7-9). Dado que la apoE juega un importante

\footnotetext{
Correspondencia:

Patricia Landázuri, Facultad de Ciencias de la Salud, Universidad del Quindío, Avenida Bolívar 12 N, Armenia, Colombia.

Teléfono: (576) 746 0158; fax: (576) 7460148.

plandazu@uniquindio.edu.co
}

Recibido: 04/08/08; aceptado:13/03/09 papel en el metabolismo de las lipoproteínas que la contienen (10), es importante estudiar de qué manera los polimorfismos en el gen de la apoproteína E pueden modificar la concentración de lípidos plasmáticos en los niños por sexo y edad. Por lo tanto, este estudio fue diseñado para evaluar la relación de los polimorfismos del gen de la apoE con el perfil lipídico en escolares del departamento del Quindío, Colombia, según edad y sexo, para identificar de manera temprana el riesgo cardiovascular en ellos, asociado a estas variables.

\section{Materiales y métodos}

\section{Diseño del estudio}

Es un estudio descriptivo de corte transversal, realizado en el año 2006, con una muestra representativa de la población escolar del departamento del Quindío, según edad, sexo, tipo de colegio y estrato socioeconómico, con un intervalo de confianza de $95 \%$ y un error de muestreo de 5\%. La selección se estableció por conglomerados de colegios públicos y privados, y se realizó mediante un muestreo aleatorio simple. Los colegios participantes tenían autorización de sus rectores y, los escolares, el consentimiento informado de los padres o adultos responsables. Este trabajo fue aprobado por el Comité de Bioética de la Universidad del Quindío.

\section{Variables}

La información demográfica básica se recolectó mediante una encuesta. Se obtuvieron $7 \mathrm{ml}$ de sangre por punción venosa en tubos secos, después de 12 horas de ayuno. El suero se obtuvo por centrifugación a $2.500 \mathrm{~g}$ por 15 minutos, a $4^{\circ} \mathrm{C}$, después de una hora de la recolección de la sangre, fue separado en microtubos y 
se almacenó a $-20^{\circ} \mathrm{C}$ hasta su uso. El mismo día de la toma de la muestra sanguínea, se determinó el peso, la talla y se cuantificaron las fracciones lipídicas. El peso corporal se midió con una balanza electrónica y se registró en kilogramos con un decimal. La estatura se midió con tallímetro y se registró con dos decimales. Cada medida se evaluó y se registró dos veces. Con el peso y la talla, se calculó el índice de masa corporal (IMC) en $\mathrm{kg} / \mathrm{m}^{2}$.

El colesterol total, los triacilglicéridos y el colesterol en las lipoproteínas de alta densidad (c-HDL), se midieron por métodos enzimáticos (estuche Sera-Pak plus, Bayer). Para quienes tenían los triglicéridos por debajo de $400 \mathrm{mg} /$ dl, el c-LDL se calculó con la ecuación de Friedewald (11). El colesterol en la lipoproteínas de muy baja densidad (c-VLDL), se determinó así: VLDL=triacilglicéridos $/ 5$.

El control interno de calidad se efectuó con los sueros de la marca Human ${ }^{\circledR}$. Se tomaron como valores de referencia de las fracciones lipídicas séricas por edad y sexo, los puntos de corte recomendados por The Nacional Cholesterol Education Program (NCEP) (12). Se evaluó el índice de Castelli (colesterol total/c-HDL), el cual está diseñado para adultos, pero varios autores $(7,13)$ lo han utilizado en niños como un buen índice de riesgo cardiovascular, aplicando los siguientes valores de riesgo de enfermedades cardiovasculares: en mujeres, $\geq 4,5$, y en varones, $\geq 5,2$ (14).

\section{Polimorfismo de apoE}

El ADN fue extraído de $5 \mathrm{ml}$ sangre completa tomada con anticoagulante. Para ello, se usó un estuche comercial de extracción (Wizard Genomics, Promega). La calidad y cantidad del ADN extraído se visualizaron en un gel de agarosa al $1 \%$ teñido con bromuro de etidio.

El polimorfismo del gen de apoE se determinó por la amplificación específica de los alelos, usando una reacción en cadena de la polimerasa (PCR). La PCR se realizó según el siguiente procedimiento: 200 a 500 ng de ADN genómico, tampón de PCR 1X, $\mathrm{MgCl}_{2} 1,5 \mathrm{mM}$, cebadores a $0,4 \mu \mathrm{M}$, dNTP $200 \mu \mathrm{M}$, dimetil sulfóxido (DMSO)
$10 \%$ y Taq polimerasa 3 unidades internacionales (UI), para un volumen total $50 \mu \mathrm{l}$. Las condiciones de amplificación fueron por cada ciclo (35 ciclos) de: $95^{\circ} \mathrm{C}$ por 1 minuto, hibridación a $60^{\circ} \mathrm{C}$ por 50 segundos y una extensión a $70^{\circ} \mathrm{C}$ por 2 minutos. Las secuencias de los cebadores usados para la amplificación fueron 5'-TAAGCTTGGCACGCCTGTCCAAGGA-3', para el cebador con sentido, y 5'-ACAGAATT CGCCCCGGCCTGGTACAC-3', para el cebador antisentido.

El producto amplificado fue de 144 pares de bases $(\mathrm{pb})$. Posteriormente, se realizó una digestión de $25 \mu \mathrm{l}$ del producto amplificado con $10 \mathrm{UI}$ de la enzima de restricción Hhal, durante 3 horas a $37^{\circ} \mathrm{C}$. Los fragmentos resultantes de la restricción se separaron mediante electroforesis vertical en gel de poliacrilamida al $12 \%$ con un voltaje constante de $45 \mathrm{~mA}$.

Los productos se visualizaron en un transiluminador de luz ultravioleta, tras tinción del gel con bromuro de etidio durante 30 minutos. Las siguientes longitudes de banda definieron los genotipos: apoE 3/3, 91 y 48 pb; apoE 2/2, $83 \mathrm{pb}$; y apoE 4/4, 91 y 72 pb. Los genotipos heterocigotos se determinaron por las siguientes longitudes de bandas: apoE 3/2, 91,83 y 48 pb; apoE, 3/4 91,72 y 48 pb; y apoE 2/4, como 91,83 y $72 \mathrm{pb}$.

\section{Análisis estadístico}

Se constituyeron tres grupos de niños de acuerdo con su edad (8 a 10, 11 a 14 y de 15 a 18 años). Para cada grupo de edad y sexo, se calcularon los promedios de las fracciones del perfil lipídico. Se efectuaron comparaciones de medias por el test de Mann-Whitney, porque las variables lipídicas no presentaron distribución normal. Se establecieron las diferencias estadísticas entre las proporciones mediante la prueba de $\mathrm{ji}$ al cuadrado $\left(\chi^{2}\right)$. Para buscar diferencias por sexo, se utilizó la prueba t de Student para muestras con varianzas desiguales. Las frecuencias de los alelos y genotipos se estimaron por conteo directo de los mismos.

Se constituyeron tres grupos de niños de acuerdo con su genotipo: grupo apoE2, formado 
por los niños portadores de los genotipos apoE 2/2+apoE 2/3; grupo apoE3, integrado por el genotipo apoE 3/3; y grupo apoE4, formado por los genotipos apoE 3/4+apoE 4/4. Se realizó la prueba de ji al cuadrado para verificar si las frecuencias observadas de los genotipos guardaban concordancia con las esperadas bajo la hipótesis de Hardy-Weinberg. Para comparar los niveles de lípidos y lipoproteínas entre los grupos de genotipos, se utilizó el análisis de varianza de una vía (ANOVA).

En las variables que se encontraron significancias entre grupos, se realizó la prueba de Bonferroni como post hoc. Todos los datos se presentan como la media \pm la desviación estándar. El nivel de significancia se estableció a $p<0,05$. El análisis estadístico se hizo con el programa SPSS (versión 11,5).

\section{Resultados}

\section{Población de estudio}

La muestra (500 escolares) para la evaluación de las variables la conformaron 249 varones $(49,8 \%)$ y 251 mujeres $(50,2 \%)$, distribuidos así: 112 escolares con edades entre 8 y 10 años (22,4\%), 205 entre 11 y 14 años (40,9\%) y 184 entre 15 y 18 años (36,7\%). El cuadro 1 muestra las características generales de los escolares distribuidos por sexo. Se encontraron diferencias significativas en la edad y el índice de masa corporal entre los sexos, y los promedios fueron de las dos variables mayores en las niñas.

\section{Distribución de los lípidos sanguíneos por edad y sexo}

Los datos del cuadro 2 resumen el comportamiento de los lípidos sanguíneos por edad y sexo de los escolares del estudio.
El análisis de los lípidos plasmáticos entre niños y niñas del mismo grupo de edad, mostró diferencias significativas de medias en el grupo de 15 a 18 años, en las variables c-HDL, $c-V L D L, y$ triacilglicéridos ( $p<0,05$ para todas). El análisis por grupos de edad y sexo mostró que el colesterol total y el c-LDL disminuyeron con la edad en niños. En niñas, sólo el colesterol total tuvo este comportamiento, mientras que el c-LDL disminuyó del primero al segundo grupo y aumentó en el tercero, sin alcanzar las cifras del primer grupo de edad. En todos los grupos de edad, ambas variables (colesterol total y c-LDL) fueron ligeramente más altas en las niñas que en los niños, con diferencias significativas para el colesterol total, pero no para el c-LDL. En niñas y niños, el c-HDL fue muy bajo comparado con los valores de referencia de The Nacional Cholesterol Education Program (12); además, desciende para ambos sexos en el grupo de 15 a 18 años; las diferencias fueron significativas entre niños y niñas de esa edad $(p<0,05)$ y entre ese grupo y los demás grupos de edad $(p<0,005)$.

El colesterol VLDL en niños tuvo el mismo comportamiento que el colesterol total y el c-LDL, y disminuyó con la edad; la diferencia fue significativa $(p<0,05)$ para todos los grupos de edad. En las niñas, las VLDL aumentaron en el grupo de 11 a 14 años, Las diferencias fueron significativas entre los grupos de edad. El c-VLDL fue más alto en los niños que en las niñas y las diferencias fueron significativas $(p<0,05)$.

La concentración de triglicéridos disminuye significativamente con la edad en los hombres $(p<0,05)$. En las mujeres tiene un aumento significativo en el grupo de 11 a 14 años $(p<0,05)$.

Cuadro 1. Características basales de la población de estudio distribuidas por sexo.

\begin{tabular}{lccc}
\hline Variable & $\begin{array}{l}\text { Niños } \\
\mathbf{n = 2 4 9}\end{array}$ & $\begin{array}{l}\text { Niñas } \\
\mathbf{n = 2 5 1}\end{array}$ & $\mathbf{P}$ \\
\hline Edad (años) & $12,9 \pm 2,9(8-18)$ & $13,4 \pm 2,8(8-18)$ & 0,065 \\
IMC kg/m² (rango) & $18,3 \pm 3,4(12,3-33,6)$ & $19,2 \pm 3,5(12,6-38,3)$ & 0,003 \\
\hline
\end{tabular}

IMC: índice de masa corporal

Todos los datos se presentan como la media \pm la desviación estándar.

$\mathrm{P}$ : diferencia significativa a $\mathrm{p}<0,05$ producto de un ANOVA 
Cuadro 2. Perfil lipídico, índice arterial e índice de masa corporal distribuidos por edad y sexo.

\begin{tabular}{|c|c|c|c|c|c|c|c|}
\hline \multirow[t]{2}{*}{ Variable } & \multicolumn{2}{|c|}{ 8-10 años } & \multicolumn{2}{|c|}{ 11-14 años } & \multicolumn{2}{|c|}{ 15-18 años } & \multirow[t]{2}{*}{$\mathbf{p}$} \\
\hline & $\begin{array}{c}\text { Niños } \\
n=60\end{array}$ & $\begin{array}{c}\text { Niñas } \\
n=52\end{array}$ & $\begin{array}{l}\text { Niños } \\
n=107\end{array}$ & $\begin{array}{c}\text { Niñas } \\
n=98\end{array}$ & $\begin{array}{c}\text { Niños } \\
n=82\end{array}$ & $\begin{array}{l}\text { Niñas } \\
n=102\end{array}$ & \\
\hline CT & $153,4 \pm 24,4$ & $154,6 \pm 33,0$ & $149,2 \pm 26,6$ & $150,8 \pm 26,0$ & $140,6 \pm 36,0$ & $148,9 \pm 33,6$ & 0,048 \\
\hline c-LDL & $91,7 \pm 26,8$ & $97,1 \pm 30,9$ & $88,2 \pm 27,1$ & $90,1 \pm 23,7$ & $86,5 \pm 35,5$ & $93,6 \pm 27,7$ & 0,304 \\
\hline c-HDL & $38,4 \pm 9,9$ & $38,0 \pm 8,3$ & $38,4 \pm 9,6$ & $38,5 \pm 8,0$ & $34,1 \pm 7,4$ & $37,1 \pm 7,9^{*}$ & 0,005 \\
\hline c-VLDL & $23,2 \pm 14,9$ & $19,4 \pm 11,5$ & $22,5 \pm 12,9$ & $22,1 \pm 10,9$ & $19,9 \pm 8,7$ & $18,1 \pm 9,8^{*}$ & 0,011 \\
\hline TAG & $116,2 \pm 74,5$ & $97,1 \pm 57,3$ & $112,9 \pm 64,7$ & $110,9 \pm 54,5$ & $99,6 \pm 43,8$ & $90,6 \pm 49,0^{*}$ & 0,011 \\
\hline CT/HDL & $4,2 \pm 1,0$ & $4,2 \pm 1,1$ & $4,1 \pm 0,9$ & $4,0 \pm 0,9$ & $4,2 \pm 1,3$ & $4,1 \pm 1,1$ & 0,280 \\
\hline IMC & $16,5 \pm 3,3$ & $16,9 \pm 1,9$ & $17,9 \pm 3,3$ & $19,0 \pm 3,6^{*}$ & $19,9 \pm 2,9$ & $21,0 \pm 2,8^{*}$ & 0,001 \\
\hline
\end{tabular}

CT: colesterol total; c-LDL: colesterol en lipoproteínas de baja densidad; C-HDL: colesterol en lipoproteínas de alta densidad; c-VLDL: colesterol en lipoproteínas de muy baja densidad; TAG: triacilglicéridos; ${ }^{*}$ :con diferencias estadísticas significativas por sexo a $p<0,05$; $p$ : significancia entre los grupo de edad.

En los tres grupos de edad, los triacilglicéridos siempre fueron mayores en los hombres comparados con las mujeres $(p<0,05)$.

Respecto al índice arterial (relación colesterol total/c-HDL), se encontró un valor promedio de 4,1 para sexos y grupo de edad, sin diferencia significativa.

\section{Índice de masa corporal}

El análisis del índice de masa corporal entre niños y niñas del mismo grupo de edad, mostró diferencias significativas de medias en el grupo de 11 a 14 y de 15 a 18 años. El análisis por grupos de edad y sexo mostró que, en ambos sexos, aumenta con la edad con diferencias significativas entre ellos $(<0,01)$. En niñas es mayor que en los niños en todos los grupos de edad $(p<0,01)$.

\section{Frecuencias alélicas y genotípicas del polimorfismo apoE}

El polimorfismo del gen de apoE se determinó en 500 escolares (249 niños y 251 niñas). La distribución genotípica y la frecuencia alélica se muestran en el cuadro 3. La distribución de los polimorfismos estuvo en equilibrio de HardyWeinberg y no se encontraron diferencias en las frecuencias entre niños y niñas. El alelo más frecuente fue el E3 $(91,6 \%)$, seguido de E2 $(5,3 \%)$ y E4 $(3,1 \%)$. En toda la población, el genotipo más común fue el E3/E3 $(90,8 \%)$, seguido del E2/E3 (5,6\%) y el E3/E4 (3,2\%). No se encontraron homocigotos para E2/E2 y
Cuadro 3. Frecuencia alélica y genotípica de los polimorfismos en el gen de la apoE en población de escolares quindianos.

\begin{tabular}{lccc}
\hline $\begin{array}{l}\text { Frecuencias } \\
\text { alélicas }\end{array}$ & $\begin{array}{c}\text { Total } \\
(\mathbf{n}=\mathbf{5 0 0 )}\end{array}$ & $\begin{array}{c}\text { Niños } \\
(\mathbf{n}=\mathbf{2 4 9})\end{array}$ & $\begin{array}{c}\text { Niñas } \\
(\mathbf{n}=\mathbf{2 5 1})\end{array}$ \\
\hline E2 & $5,3(29)$ & $5,2(14)$ & $5,5(15)$ \\
E3 & $91,6(499)$ & $92,0(249)$ & $91,6(250)$ \\
E4 & $3,1(17)$ & $3,0(8)$ & $3,3(9)$ \\
Frecuencias & genotípicas & & \\
E2/E2 & 0 & 0 & 0 \\
E2/E3 & $5,6(28)$ & $5,6(14)$ & $5,6(14)$ \\
E2/E4 & $0,2(1)$ & 0 & $0,4(1)$ \\
E3/E3 & $90,8(455)$ & $91,2(227)$ & $90,8(228)$ \\
E3/E4 & $3,2(16)$ & $3,2(8)$ & $3,2(8)$ \\
E4/E4 & 0 & 0 & 0 \\
\hline
\end{tabular}

E4/E4. Similar distribución genotípica y alélica se encontraron para cada uno de los sexos (cuadro 3).

\section{Distribución del polimorfismo de la apoE y de los lípidos sanguíneos por sexo}

La relación entre genotipos y lípidos en niños y niñas se presenta en el cuadro 4. La niña E2/ E4 fue eliminada de los análisis debido al efecto opuesto de sus dos alelos sobre los lípidos. Una niña E2/E3 fue eliminada del grupo por su valores de lípidos en niveles patológicos (colesterol total $=306,5, c-L D L=179,0$ y triacilglicéridos $=410$ $\mathrm{mg} / \mathrm{dl})$.

Los datos muestran que el tipo de alelo apoE tiene un efecto significativo $(p<0,05)$ sobre el colesterol total, el c-LDL, y el índice de Castelli (colesterol total/c-HDL) en cada sexo y entre sexos. Así, en los niños, los niveles de colesterol 
Cuadro 4. Distribución del polimorfismo de la apoE y de los lípidos sanguíneos por sexo.

\begin{tabular}{|c|c|c|c|c|}
\hline & $\begin{array}{c}\text { E2 } \\
\text { (13 niñas, } \\
14 \text { niños) }\end{array}$ & $\begin{array}{c}\text { E3 } \\
\text { (228 niñas, } \\
227 \text { niños) }\end{array}$ & $\begin{array}{c}\text { E4 } \\
\text { (8 niñas, } \\
8 \text { niños) }\end{array}$ & $\mathbf{P}$ \\
\hline \multicolumn{5}{|l|}{ CT } \\
\hline Niños & $134,3(91,7-178,5)$ & $147,1(90-351)$ & $180,5(144,5-221)$ & \\
\hline $\begin{array}{l}\text { Niñas } \\
\text { c-LDL }\end{array}$ & $142,4(109-194,0)$ & $150,4(5,1-241)$ & $149,6(169-106)^{*}$ & 0,015 \\
\hline Niños & $74,5(37,4-111,1)$ & $88,6(11,7-298)$ & $116,9(91,8-146,7)$ & \\
\hline $\begin{array}{l}\text { Niñas } \\
\text { c-HDL }\end{array}$ & $82,5(33,6-125,8)$ & $92,9(371-83,4)^{*}$ & $89,5(114,5-62,6)^{*}$ & 0,005 \\
\hline Niños & $39,1(55,5-25,5)$ & $36,8(22-72)$ & $38,8(60,2-21,7)$ & \\
\hline $\begin{array}{l}\text { Niñas } \\
\text { c-VLDL }\end{array}$ & $40,8(25,6-55,0)$ & $37,6(22-65,5)$ & $43,6(56,7-30)$ & 0,290 \\
\hline Niños & $23,7(10-42)$ & $21,7(6,3-76.8)$ & $24,8(46,4-13,2)$ & \\
\hline $\begin{array}{l}\text { Niñas } \\
\text { TAG }\end{array}$ & $19,1(6,8-31,5)$ & $19,9(62-6,8)^{*}$ & $16,3(32,5-5,6$ & 0,727 \\
\hline Niños & $118,7(50-210)$ & $108,3(384-31,5)$ & $123,9(232,2-66)$ & \\
\hline $\begin{array}{l}\text { Niñas } \\
\text { CT /HDL }\end{array}$ & $95,3(34-162,3)$ & $99,3(310-34)^{*}$ & $81,4(162,5-28)$ & 0,727 \\
\hline Niños & $3,6(2,3-7)$ & $4,2(9,2-2)$ & $4,9(5-3,7)$ & \\
\hline Niñas & $3,6(2,9-5,6)$ & $4,1(8,5-2,2)$ & $3,5(4,1-2,9)^{*}$ & 0,006 \\
\hline
\end{tabular}

${ }^{*}$ Con diferencias estadísticas significativas a $\mathrm{p}<0,05$ en cada variable comparadas por sexo. Los valores son la media (rango); $P$, significativo entre los alelos sin diferenciar sexo.

CT: colesterol total; c-LDL: colesterol en lipoproteínas de baja densidad; c-HDL: colesterol en lipoproteínas de alta densidad; c-VLDL: colesterol en lipoproteínas de muy baja densidad; TAG: triacilglicéridos

total y c-LDL se distribuyeron por genotipo de la siguiente manera: $E 4>E 3>E 2(p<0,05)$; mientras que, en las niñas, ocurre lo contrario. Los niveles de colesterol total y c-LDL se distribuyeron así: E3>E4>E2. Para los niveles de c-HDL, la distribución en niños fue así: E2>E4>E3. Por el contrario, en las niñas, el alelo E4 presentó mayores niveles de c-HDL; la distribución fue así: E4>E2>E3. En cualquier polimorfismo, los hombres tienen niveles bajos de c-HDL comparados con los niveles de referencia.

En los niños, los niveles de c-VLDL y de triacilglicéridos se distribuyeron así, E4>E2>E3 $(p<0,05)$; mientras que, en las niñas, el c-VLDL y los triacilglicéridos se distribuyeron así: E3>E2>E4, $(p<0,05)$. La relación colesterol total/ HDL fue estadísticamente significativa para el tipo de alelo y para el sexo ( $p<0,05$ para ambos); para los niños, la distribución por genotipos fue E4>E3>E2 y en las niñas fue E3>E2>E4.

En resumen, el alelo de riesgo en los niños fue el alelo E4, el cual presentó colesterol total, c-LDL, triacilglicéridos y c-VLDL más altos que los niños con los otros dos alelos. El alelo E2 fue el alelo protector, con los valores más bajos para las mismas variables. Por el contrario, en las niñas el alelo de riesgo fue el E3, el cual presentó concentraciones de colesterol total, c-LDL, triacilglicéridos y c-VLDL más altos que en las portadoras de los otros dos alelos. El alelo E4 podría ser protector pues presentó los valores más bajos de triacilglicéridos y c-VLDL $y$ valores intermedios para colesterol total y c-LDL. En el mismo sentido, los niveles de c-HDL tuvieron expresión diferente con respecto al sexo, mientras que el alelo E2 tenía las concentraciones de c-HDL más altas en niños y, el E4, las más bajas. En las niñas, el E3 fue el de menor valor y el E4 el más alto.

\section{Discusión}

Recientes estudios han mostrado que las dislipidemias también son un problema de la población joven, razón por la cual, muchos esfuerzos en investigación se han focalizado en describir la prevalencia de estas dislipidemias y sus causas y consecuencias en la infancia (15-17). 
En relación con el polimorfismo apoE, varios estudios nacionales (18-20) e internacionales $(1,2)$ se han enfocado en la interacción de este polimorfismo con los lípidos plasmáticos. En Colombia, éste es uno de los pocos trabajos que hay de genotipificación del polimorfismo de la apoE y su relación con los lípidos sanguíneos en escolares aparentemente sanos. En relación con las frecuencias, los resultados muestran que en esta población no se encontró diferencia entre los valores observados de las frecuencias alélicas y genotípicas y los valores esperados, de tal manera que la población estaba en equilibrio de Hardy-Weinberg. Esto podría significar que la población estudiada no ha sido sometida a migraciones de población significativas de diferentes orígenes y mezclas recientes, 0 que la migración del campo a la ciudad quedó solapada porque el grupo incluye escolares de origen urbano y rural.

Por otro lado, el análisis muestra que la distribución del alelo E3 y el genotipo E3E3, fue más alta $(90,8 \%$ y $91,6 \%$, respectivamente) que la encontrada para el mismo alelo en Venezuela (83\%) (21), Corea (85,2\%) (22) y Grecia $(80,7 \%)$ (23), muy cercana a la encontrada en una población adulta en Bogotá, Colombia (87\%) (19) y en una población de escolares del centro oriente colombiano (86\%) (20). La distribución fue similar a la descrita en un grupo de adolescentes mexicanos (89,5\%) (24), una población indígena mexicana $(90 \%)$ y una población mestiza mexicana $(91,5 \%)$ (25); en estas dos últimas poblaciones, no se encontraron homocigotos E2 y E4, igual a los hallazgos de este estudio. Esto sugiere una influencia racial en la distribución genotípica y alélica en la población quindiana. La ausencia de homocigotos E2 y E4 en esta población, es diferente a los resultados encontrados en una población de jóvenes finlandeses, donde el genotipo E4E4 fue de 3,2\% (26); y fue muy similar a los hallazgos en algunos grupos de población colombianos del centro y del centrooriente del país, donde las frecuencias de los genotipos homocigotos E4 y E2 fueron muy bajas (E4E4 1\%, E2E2 0,3\%) (18-20). De hecho, se conoce que la población quindiana pertenece a la autodenominada "comunidad paisa", en la cual se ha estimado que el componente racial ancestral es $85 \%$ caucásica y $15 \%$ amerindia $(27,28)$. Los datos del estudio sugieren que, para esta población joven, la influencia indígena puede ser importante en la distribución genotípica del polimorfismo de la apoE. Sin embargo, es necesario profundizar más en este aspecto racial.

Además, este estudio muestra que el polimorfismo apoE tiene un efecto significativo sobre los niveles de colesterol total y c-LDL plasmáticos de los escolares quindianos. En los varones, el colesterol total y el c-LDL se distribuyeron de acuerdo con lo descrito en otras poblaciones $(1,21)$ y en otros grupos de la población colombiana (18-20), en los cuales se muestran bajos niveles de colesterol total y c-LDL y altos niveles de c-HDL, asociados al alelo E2, lo que sugiere un efecto protector antiaterogénico relacionado con ese alelo, contrario al efecto aterogénico en los portadores del alelo $\mathrm{E} 4$, el cual está frecuentemente asociado con altos niveles de colesterol total y c-LDL y bajos niveles de c-HDL. Sin embargo, en las niñas el efecto fue diferente con respecto al alelo E4; las portadoras del mismo tenían niveles intermedios de los lípidos aterogénicos (colesterol total y c-LDL), los niveles más bajos de triacilglicéridos y c-VLDLy, los mayores niveles de c-HDL. El alelo E3 tenía los niveles más altos de colesterol total y c-LDL, y los más bajos de c-HDL. Estos resultados sugieren una diferencia de sexo sobre la influencia del polimorfismo de la apoE en los lípidos plasmáticos de esta población a edades tempranas.

Estas diferencias de sexo en los niveles de lípidos dentro de cada genotipo apoE, se han descrito en otros estudios de población $(29,30)$. Al respecto, varios de ellos han reportado la influencia de las hormonas sexuales, principalmente los estrógenos, en la modulación de la concentración de los lípidos plasmáticos por la apoE. Schaefer et al. (29) mostraron diferencias en los niveles plasmáticos de c-LDL en cada fenotipo de apoE, entre hombres jóvenes ( $<53$ años) y mayores ( $>53$ años), pero en las mujeres estas diferencias se limitaron 
sólo a las posmenopáusicas. Otros estudios han mostrado que las hormonas sexuales modulan el impacto del apoE en el metabolismo de las lipoproteínas, de tal modo, que la expresión de alteraciones en los lípidos es más frecuente en hombres que en mujeres y que, cuando estos ocurren en mujeres, son más frecuentes en la posmenopáusicas (31).

Sin embargo, nuestros datos no sólo muestran diferencias de sexo en la concentración de lípidos en cada genotipo, sino, en la isoforma apoE que los expresa; por ejemplo, como se mencionó antes, los resultados muestran que en las niñas, para colesterol total y c-LDL, el efecto protector está en el alelo E2, pero, para triacilglicéridos, c-VLDL y c-HDL, el efecto protector está en el alelo E4. Es posible que la discrepancia encontrada aquí con respecto a los estudios de población descritos, se deba a dos razones; en ellos, no se hicieron distribuciones simultáneas por grupos de edad y sexo o los grupos estudiados incluían sólo poblaciones adultas, o sólo poblaciones jóvenes, estas últimas con edades fisiológicamente más homogéneas, en las cuales posiblemente no había cambios hormonales importantes, como son el inicio y final de la pubertad. Por el contrario, el presente estudio incluye escolares en edades prepuberales (8 a 10 años) y escolares en el inicio y final de la pubertad (11 a 18 años), en quienes la producción de hormonas sexuales es el evento fisiológico más importante de esa etapa de la vida. Estos cambios fisiológicos podrían explicar, en parte, por qué los resultados analizados por sexo muestran diferencias en la influencia del genotipo apoE en los lípidos sanguíneos. Estos datos son concordantes con lo descrito por Garcés et al. (32), en cuanto a la influencia del sexo en una población de niños prepúberes (6 a 7 años). El estudio de Garcés mostró que el porcentaje en el que cambian los lípidos sanguíneos (colesterol total y c-LDL) fue diferente entre niños y niñas y dependió del genotipo. Además de esto, el mismo estudio sugirió una asociación entre la hormona androgénica de la suprarrenal, el sulfato de dehidroepiandrosterona (DHEA-S), y el efecto que tiene el polimorfismo apoE sobre los lípidos sanguíneos. Esta asociación mostró que el DHEA-S mejoró el efecto hipolipemiante del alelo E2 y disminuyó el efecto hiperlipémico del E4 (32). Esta relación entre el DHEA-S y el genotipo podría explicar, de alguna manera, por qué en las niñas de este estudio el alelo E4 presentaba los niveles de colesterol total y c-LDL más bajos.

La DHEA y el DHEA-S son andrógenos débiles pero muy abundantes; además, son los principales andrógenos en las niñas y se ha demostrado que aumentan con la edad en hombres y mujeres, y declinan en ambos sexos hacia la tercera década (33). Estas variaciones podrían determinar su influencia sobre los lípidos en las diferentes etapas de la vida de las mujeres, incluidas las niñas de nuestra población de estudio.

Además de la producción de hormonas sexuales suprarrenales, niños y niñas entre los 10 y 11 años (con algunas diferencias de sexo), comienzan la maduración gonadal y la producción de hormonas sexuales gonadales a partir del colesterol. Como consecuencia de la diferencia cronológica en la maduración sexual, los niveles de hormonas sexuales gonadales y la DHEA podrían explicar la variación en la influencia del polimorfismo de la apoE con la edad sobre los niveles sanguíneos de lípidos de esta población, específicamente en las niñas. Al respecto, se ha demostrado que la apoE determina los niveles de testosterona y DHEA, posiblemente, modulando el aporte de colesterol para la síntesis de estas hormonas (34). Otra variable importante y determinante en el nivel de lípidos y hormonas es el IMC. Varios estudios han demostrado que esta variable conserva una relación directa con el colesterol total e una inversa con las HDL (35). En este trabajo, las niñas entre 11 y 18 años tienen un índice de masa corporal significativamente mayor que los niños de la misma edad y también el colesterol total, lo que prueba esta relación. Sin embargo, la estratificación de índice de masa corporal por genotipos (datos no mostrados) no arroja resultados claros que pudieran aportar a la discusión. Todos estos hechos, edad, género, índice de masa corporal, diferencias en la 
producción de hormonas sexuales y el efecto de la apoE en la captación de colesterol por las células, podrían explicar en parte las diferencias entre niños y niñas aquí descritas y, sobre todo, entre los grupos de edad de las niñas. Ssin embargo, se requiere de más estudios que precisen el aporte de cada una de estas variables de confusión que complica el análisis de la influencia del genotipo apoE en los lípidos plasmáticos.

Indudablemente, la influencia del sexo y la edad en la modulación de los lípidos por las isoformas de la apoE, está demostrada en ésta y en otras poblaciones, y estos hallazgos sugieren, más no prueban, un papel importante de las hormonas sexuales en las diferencias de expresión de los lípidos relacionadas con el genotipo de la apoE. Dado que este trabajo no accedió a los niveles hormonales de la población de estudio para corroborar dicha influencia, el análisis de los resultados es limitado y sólo sugestivo, pero plantea la necesidad de hacer estudios que relacionen estos parámetros en la población, para fundamentar un manejo futuro, individual, por sexo y edad, de las enfermedades relacionadas con los lípidos plasmáticos.

En conclusión, los datos del estudio sugieren una asociación entre los niveles de lípidos plasmáticos de los escolares estudiados y los polimorfismos de la apoE. Esta asociación es influenciada por el sexo y la edad, y esto es más notorio en las niñas mayores de 10 años, en quienes se inicia un cambio fisiológico importante como es la pubertad.

Además, el estudio sugiere una influencia racial en la distribución alélica y genotípica de la apoE que requiere de profundización.

\section{Agradecimientos}

A los colegios públicos y privados que apoyaron la realización del trabajo, a los padres y niños participantes.

\section{Conflicto de interés}

Los autores manifiestan que no existen conflictos de intereses en la elaboración y ejecución de este proyecto.

\section{Financiación}

Universidad del Quindío, código 318.

\section{Referencias}

1. Hixson JE. Apolipoprotein E polymorphism affect atheroesclerosis en young males. Pathobiological determinants of atheroesclerosis in Youth (PDAY) Research Group. Arterioescler Thromb. 1991;11:1237-44.

2. Heeren J, Beisiegel U, Grewal T. Apolipoprotein E recycling: Implications for dyslipidemia and atherosclerosis. Arterioscler Thromb Vasc Biol. 2006;26;442-8.

3. Bennet AM, Di Angelantonio E, Ye Z, Wensley F, Dahlin A, Ahlbom A, et al. Association of apolipoprotein E genotypes with lipid levels and coronary risk. JAMA. 2007;298:1300-11.

4. Dong LM, Wilson C, Wardell MR, Simmons T, Mahley $\mathbf{R}$, Weisgraber $\mathbf{K H}$, et al. Human apolipoprotein E: Role of arginine 61 in mediating the lipoprotein preferences of the E3 and E4 isoforms. J Biol Chem. 1994;269:2235865.

5. Ministerio de la Protección Social, Organización Panamericana de la Salud. Situación de Salud Colombia. Indicadores básicos 2007. p. 24. Fecha de consulta: 13 de septiembre de 2008. Disponible en: http://www.minproteccionsocial.gov.co/vBecontent/ NewsDetail.asp?ID=15895\&IDCompany=3

6. Instituto Seccional de Salud del Quindío. Indicadores básicos en salud. SIVIGILA. Bogotá D.C.: INS; 2005.

7. Uscátegui RM, Álvares MC, Leguado I, Soler W, Martínez I, Arias R, et al. Factores de riesgo cardiovascular en niños de 6 a 18 años de Medellín, Colombia. An Pediat (Barc). 2004:58:411-7.

8. Gracia B, de Plata C. Pradilla A, Leiva J. Factores de riesgo para enfermedades de mayor prevalencia en el Valle del Cauca útiles para el desarrollo de estrategias de prevención. Colomb Med. 2003;34:47-55.

9. Poveda E, Callas N, Baracaldo C, Castillo C, Hernández P, Guerra M. Evaluación de las concentraciones de lípidos y apoproteínas A-I y B-100 en un grupo de escolares de cinco departamentos del centro-oriente de Colombia. Biomédica. 2007;27:385-99.

10. Song Y,Stampfer MJ, Liu S. Meta-analysis: apolipoprotein E genotypes and risk for coronary heart disease. Ann Intern Med. 2004;141:137-47.

11. Friedewald WT, Levy RI, Fredickson DS. Estimation of the concentration of c-LDL in plasma without use of the preparative ultracentrifuge. Clin Chem. 1972;18:499502.

12. Expert Panel on Detection, Evaluation, and Treatment of High Blood Cholesterol in Adults. Executive summary of the Third Report of National Cholesterol 
Education Program (NCEP). Expert Panel on Detection. Evaluation, and Treatment of High Blood Cholesterol in Adults (Adult treatment Panel III). JAMA. 2001;285:248696.

13. Scherr C, Magalhães CK, Malheiros W. Lipid profile analysis in school children. Arq Bras Cardiol. 2007;89: 65-70.

14. Castelli W, Fernández-Cruz A. Recomendaciones de ILIB para el diagnóstico de las dislipidemias en Latinoamérica. Cardiovas Risk Fact (edición en español). 1994;3(Suppl.1):10-27.

15. Kavey RE, Daniels SR, Lauer RM, Atkins DL, Hayman LL, Tauber K. American Heart Association guidelines for primary prevention of atherosclerotic cardiovascular disease beginning in childhood. J Pediatr. 2003; 142: 368-72.

16. Ford ES, Mokdad AH, Ajani UA. Trends in risk factors for cardiovascular disease among children and adolescents in the United States. Pediatrics. 2004;114:1534-44.

17. Kavey RE, Daniels SR, Lauer RM, Atkins DL, Hayman LL, Taubert K. American Heart Association guidelines for primary prevention of atherosclerotic cardiovascular disease beginning in childhood. Circulation. 2003;107:1562-6.

18. Moreno A, Torres AL, Cartagena AE, Herrera MH. Determinación de la asociación del polimorfismo de la apolipoproteína $\mathrm{E}$ (Apo E) relacionada con la presencia de placa aterosclerótica coronaria. NOVA. 2006;4.1-116.

19. Torres AL, Guerra-Muñoz M, Segrera A, Wagner J, Alvarado M. Modulación de los niveles de lípidos y lipoproteínas por el polimorfismo de la apolipoproteína E en individuos sanos de Bogotá D.C. NOVA. 2005; 3:1-120.

20. Callas N, Poveda E, Baracaldo C, Hernández P, Castillo C, Guerra M. Polimorfismo genético de la apolipoproteína $\mathrm{E}$ en un grupo de escolares del centrooriente colombiano:comparación con las concentraciones plasmáticas de lípidos y apolipoproteínas. Biomédica. 2007;27:526-36

21. Celaya J, Rodríguez A, Michelle P, Arends A. Estudios de polimorfismos del gen (APOE) de la apolipoproteína-E (Apo E) y su relación con niveles elevados de colesterol total, lipoproteínas y triglicéridos séricos en niños de edad escolar. Rev Soc Med Quir Hosp Emerg Perez de León. 2007;38(Suppl.1):19-26.

22. Shin MH, Kim HN, Cui LH, Kweon SS, Park KS, Heo $\mathbf{H}$, et al. The effect of apolipoprotein $\mathrm{E}$ polymorphism on lipid levels in Korean adults. J Korean Med Sci. 2005;20:361-6.

23. Liberopoulos E, Miltiadous G, Hatzivassiliou M, Ayrton N, Bairaktari E, Cariolou M, et al. Apolipoprotein E polymorphism in northwestern Greece: Frequency and effect on lipid parameters. Ann Clin Lab Sci. 2004; 34:347-54.

24. Medina-Urrutia AX, Cardoso-Saldana GC, ZamoraGonzález J, Liria YK, Posadas-Romero C. Apolipoprotein $\mathrm{E}$ polymorphism is related to plasma lipids and apolipoprotein in Mexican adolescents. Hum Biol. 2004;76:605-14.

25. Gamboa R, Hernández-Pacheco G, Hesiquio R, Zúniga J, Masso F, Montano LF, et al. Apolipoprotein E polymorphism in the Indian and Mestizo populations of Mexico. Hum Biol. 2000;72:975-81.

26. Lehtimaki T, Moilanen T, Viikari J, Herblom HK, Ehnholm C, Riinnemaa T, et al. Apolipoprotein E phenotypes in Finnish youths: a cross-sectional and 6-year follow-up study. J Lipid Res. 1990;31:487-95.

27. Parson JJ. Antioqueño colonization in western Colombia. Berkeley: University of California Press; 1949.

28. Jiménez I, Mora O, López G, Jiménez ME, Zuluaga L, Isaza R, et al. Idiopathic epilepsy with generalized tonic clonic seizures in Antioquia, Colombia: Is the joint Amerindian and Negroid racial admixture the cause of its high prevalence? Biol Res. 1996;29:297-304.

29. Schaefer EJ, Lamon-Fava S, Johnson S, Ordovas JM, Schaefer MM, Castelli WP, et al. Effects of gender and menopausal status on the association of apolipoprotein E phenotype with plasma lipoprotein levels. Results from the Framingham Offspring Study. Arterioscler Thromb. 1994;14:1105-13

30. Ortega H, Castilla P, Gómez-Coronado D, Garcés C, Benavente $\mathbf{M}$, Rodríguez-Artalejo $\mathbf{F}$, et al. Influence of apolipoprotein $\mathrm{E}$ genotype on fat-soluble plasma antioxidants in Spanish children1. Am J Clin Nutr. 2005;81:624-32.

31. Zofkova I, Zajickova K, Hill M, Horinek A. Apolipoprotein $\mathrm{E}$ gene determines serum testosterone and dehydroepiandrosterone levels in postmenopausal women. Eur J Endocrinol. 2002;147:503-6.

32. Garcés C, Benavente M, Cano B, Viturro E, Ortega $H$, Horcajada C, et al. Effects of dehidroepiandrosteronesulfate on the Apo $\mathrm{E}$ genotype influence on plasma lipid levels in prepuberal children. J Clin Endocrinol Metab. 2003;88:3997-4000.

33. Vermeulen A. Dehydroepiandrosterone sulfate and ageing. Ann NY Acad Sci. 1995;774:121-7.

34. Thorngate FE, Strockbine PA, Erickson SK. Williams DL. Altered adrenal gland cholesterol metabolism in the apoE-deficient Mouse. J Lipid Res. 2002;43:1920-6.

35. Agirbasli M, Ciliv G, Cakir S, Srinivasan S, Berenson GS, Ozme S. Body mass index and lipid levels in children from Ankara, Turkey versus Bogalusa, Louisiana. Prev Med. 2005;41:843-5. 